Original Article

\title{
One session of motor control exercise improves joint position sense assessed by an iPhone application: a randomized controlled trial
}

\author{
Peemongkon Wattananon, PT, PhD ${ }^{1)^{*}}$, Wanalee Klomjai, PT, PhD ${ }^{1)}$, Won Sung, DPT, PhD 2 ) \\ 1) Faculty of Physical Therapy, Mahidol University: 999 Phuttamonthon 4 Road, Salaya, \\ Nakhon Pathom 73170, Thailand \\ 2) Good Shepherd Penn Partners, Penn Therapy and Fitness, USA
}

\begin{abstract}
Purpose] To establish the test-retest reliability of an iPhone application and determine the immediate effect of motor control exercise (MCE) on lumbar position sense. [Participants and Methods] This study used a two-arm, randomized controlled trial design with a blinded assessor. Sixty healthy participants were randomized into the exercise or control group. The exercise group underwent 30-min MCE, whereas the control group rested for $15 \mathrm{~min}$. Lumbar motion measured by two iPhones with goniometer application was used to determine the testretest reliability. Absolute repositioning errors (pre- and post-test) from the control and exercise groups were used to determine the immediate effect of MCE on lumbar position sense. [Results] The test-retest reliability was 0.67-0.95. A significant interaction effect was found for Angle*Time, main effect of Angle, and main effect of Time. Post-hoc comparison showed a significant improvement in position sense at $45^{\circ}$ and $60^{\circ}$ in the exercise group. [Conclusion] The findings suggest that a mobile phone application has the ability to detect changes in lumbar position sense between sessions that exceed measurement error following MCE. One session of specific MCE can improve lumbar position sense at high lumbar flexion.

Key words: Proprioception, Motor control, Test-retest reliability
\end{abstract}

(This article was submitted Mar. 12, 2019, and was accepted Apr. 22, 2019)

\section{INTRODUCTION}

Proprioception has two components: sense of stationary position (position sense) and sense of movement (kinesthesia) ${ }^{1)}$. Proprioceptive impairments have been identified across many individuals with low back pain (LBP) ${ }^{2-6)}$. The inability to reposition the spine has been identified in patients with LBP and is considered to be suggestive of proprioceptive impairments ${ }^{4}$. Impaired proprioception of the lumbar spine may alter somatosensory control of the spine, resulting in recurrent to chronic LBP in individuals ${ }^{7}$.

Forward bending quality has been identified to predict those that would benefit from motor control exercises (MCEs) ${ }^{8-10)}$. Aberrant movements have been identified in the midrange of motion, where muscle control is important for spinal stability ${ }^{11)}$. Sagittal plane movement and position sense have also been identified as valuable indicators in subgrouping patients into appropriate motor control subgroups ${ }^{8)}$. Lumbar position and lordosis also have an impact on muscle activity, and repositioning errors may prevent patients from obtaining optimal spinal positions during movement ${ }^{12}$. Due to clinical utility of forward bending, repositioning errors during forward bending may provide valuable information to clinicians on patient movement control and a physical performance outcome in response to intervention.

Evidence on MCE and repositioning errors has been conflicting. Boucher et al. investigated lumbar motion sense quantified by motion perception threshold (minimal axial lumbar rotation that an individual could identify) ${ }^{13)}$. They reported that

*Corresponding author. Peemongkon Wattananon (E-mail: peemongkon.wat@mahidol.ac.th)

(C2019 The Society of Physical Therapy Science. Published by IPEC Inc.

(c) (1) $($ This is an open-access article distributed under the terms of the Creative Commons Attribution Non-Commercial No Derivatives

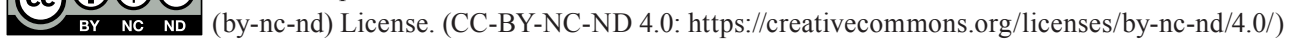


lumbar motion sense remained unchanged following an 8-week MCE. However, they acknowledged that their measurement of lumbar motion sense may solely assess passive structure (i.e., ligaments and joint capsules) ${ }^{7)}$. Kong et al. investigated the effect of an 8-week prone bridge exercise on lumbar position sense ${ }^{14}$. The result showed reduction in repositioning error during lumbar flexion and extension, indicating improvement in lumbar position sense in patients with chronic LBP. Motor function of the spine seems to require specific training during intervention ${ }^{15}{ }^{16}$. Therefore, a motor control paradigm may be better for improving position sense.

MCE is based on motor-learning concepts to restore precise co-contraction pattern of deep abdominal and back mus$\operatorname{cles}^{17,18)}$. MCE a widely used physical therapy intervention to treat patients with LBP to improve motor control ${ }^{8,18)}$. MCE typically involves three stages. The first stage emphasizes co-contraction of transverse abdominis and lumbar multifidus muscles. The second stage focuses on limb movement (self-perturbation) with co-contraction of those muscles. The last stage is incorporating co-contraction with daily activities ${ }^{18}$. Several researchers have investigated the effect of MCE on clinical and biomechanical outcomes ${ }^{19,20)}$. Improvements in muscle activation patterns and onset timing response may result in part from improved lumbar proprioception ${ }^{19,20)}$.

Several studies demonstrated immediate effect of motor control training on peripheral joint proprioception ${ }^{6,21,22)}$. They suggested that this immediate improvement may result from repetitive intentional movement that enhances afferent signals from the muscle spindle ${ }^{6,21}$. Although these studies investigated peripheral joint position sense, their finding could be applied to lumbar position sense when using the same principle. Therefore, lumbar position sense should be improved after one session of MCE based on the motor-learning concept.

However, studies related to lumbar position sense were conducted in a laboratory using high-precision equipment that limits the generalizability of those studies ${ }^{13}{ }^{14}$ ). iPhone applications can be utilized to measure the angular displacement by mounting the iPhone on the surface of a rigid body ${ }^{23}$ 24). iPhones have built-in accelerometer and gyroscope that allow the measurement of joint movement with moderate to excellent reliability ${ }^{23}{ }^{24}$. In addition, the cost of using this instrument is relatively low compared with those laboratory-based equipment. Therefore, the ability to measure joint movement using a phone application may provide a pragmatic method to measure lumbar position sense in clinical settings. In these situations, dedicated laboratory instruments may not be accessible or practical, making phone applications a more suitable option in measuring lumbar position sense.

Therefore, the purposes of the present study were to 1) determine the test-retest reliability of an iPhone application measurement and protocol and 2) determine the immediate effect of MCE on lumbar position sense in healthy individuals at difference angles. We hypothesized that the test-retest reliability would be excellent, and healthy individuals who underwent MCE would demonstrate significantly greater improvement in lumbar position sense. The results of this study would provide a method to measure lumbar repositioning sense with reliability and sensitivity to change for these values. It also provides methodology for obtaining objective measures of improvement using clinically useful tools in future studies of individuals with LBP.

\section{PARTICIPANTS AND METHODS}

This study was a randomized controlled trial using mixed design with two repeated measures (time: pre- and postexercise; angle: $30^{\circ}, 45^{\circ}$, and $60^{\circ}$ ) and one independent measure (control and exercise groups). The assessor was blinded to the group assignment. Our study protocol was approved by the university institutional review board (COA No. MU-CIRB 2016/047.0704) and registered to clinicaltrial.gov (NCT02974062). All participants provided written informed consent before study participation. The present study was conducted at the Faculty of Physical Therapy, Mahidol University, Nakhon Pathom, Thailand. Data were collected from April to May 2016.

Sixty participants were recruited from a sample of convenience from the university community. The inclusion criteria included no episode of LBP, and no regular exercise routine was composed of core exercise. Participants were excluded if they had clinical signs of systemic disease, definitive neurologic signs, previous spinal surgery, inflammatory joint disease, vestibular dysfunction, or body mass index (BMI) of $>30 \mathrm{~kg} / \mathrm{m}^{2}$. All participants provided a written informed consent before the data collection process. Preliminary repositioning sense data on 10 participants following MCE was used to calculate the sample size ( $\mathrm{G}^{*}$ Power version 3.1.9.2, University Kiel, Germany) by using a moderate effect size (0.53) at a confidence level $(\alpha)$ of 0.05 and a power $(1-\beta)$ of 0.80 . Therefore, approximately 30 participants were required for each group (exercise and control).

Two iPhone model 5S with iOS 10 (iPhone ${ }^{\circledR}$ is a trademark of Apple Inc., Cupertino, CA, USA) and iPhone application (Goniometer Pro version 2.7, 5fuf5 Co., Bloomfield, NJ, USA) were used in this study. Data from the iPhone application were used to determine the test-retest reliability of the iPhone application measurement and protocol and establish a standard error of measurement (SEM), as well as determine the immediate effect of MCE on lumbar position sense by measurement of absolute repositioning errors at $30^{\circ}, 45^{\circ}$, and $60^{\circ}$ pre- and post-exercise. Theoretically, greater repositioning error would represent greater impairment of lumbar position sense ${ }^{25,26)}$. In addition, using an iPhone application is more applicable for clinical practice.

The overall study scheme is presented in Fig. 1A. After the participants provided written informed consent, they completed a questionnaire for demographic data. Body landmarks, including lumbar spine (L1) and pelvis (S2), were identi- 


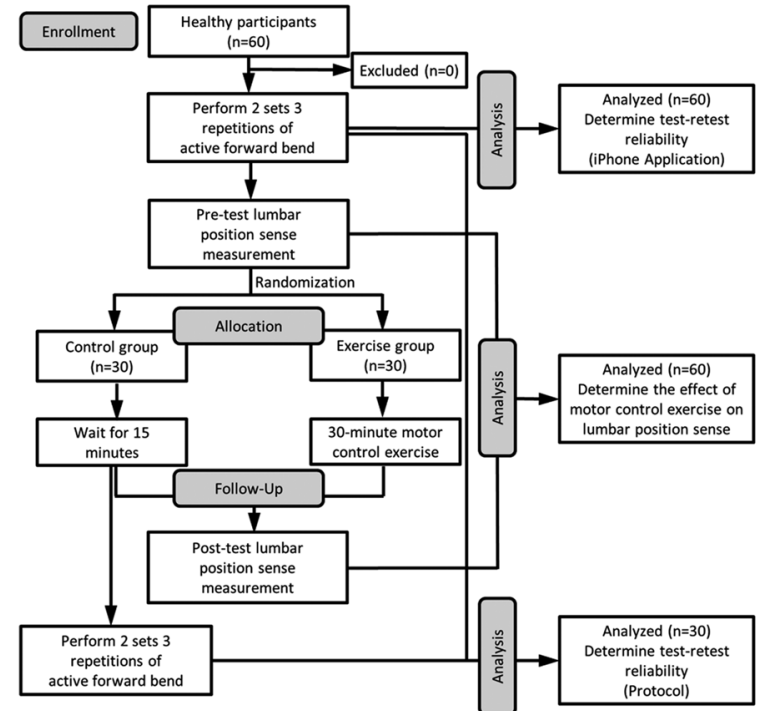

(A)

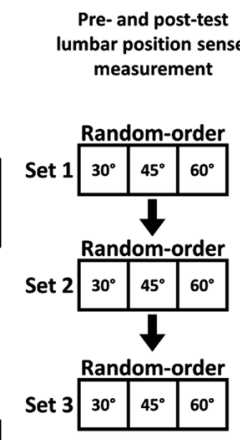

(B)

Fig. 1. CONSORT flow diagram representing the overall study scheme (A) and detailed pre- and post-test lumbar position sense measurement design (B).

fied and marked with a skin pen for iPhone placement (Fig. 2A). Each iPhone was placed in the iPhone pocket with transparent film to visualize the iPhone monitor. The pockets were attached to the participant by using a Velcro strap around his/her trunk and pelvis (the lower border of the iPhones was aligned with spinous processes). The data collection process started after the participants had completed the set up. Investigators who collected data were blinded to the group assignment.

The participants stood on a drawing paper with feetshoulder width apart. The foot print was drawn using a marker. This foot print was used for post-test positioning. The participants stood in a natural stance with arms down on the sides. The participants were instructed to stand up straight and maintain that position to record lumbar and sacral angles in the neutral position. After recording, the participants were instructed to perform two sets of three repetitions of standing forward and bend as far as possible and return to the upright position (Fig. 2B and C). Lumbar and pelvic angles at full trunk flexion, as well as lumbar and pelvic angles at completion of returning to upright were recorded for each repetition. Data for each set were used to determine the test-retest (intra-trial) reliability of an iPhone application, as well as establish the SEM (intra-trial).

The participants performed practice trials of trunk flexion at $30^{\circ}, 45^{\circ}$, and $60^{\circ}$ (four repetitions for each degree) with verbal feedback. These practice trials aimed for participants to familiarize with the testing protocol, as well as minimize the learning effect. After completion of practice trials, participants underwent pre-test data collection by performing three sets of trunk flexion at random-order angles $\left(30^{\circ}, 45^{\circ}\right.$, and $\left.60^{\circ}\right)$ without feedback (Fig. 1B). Investigators recorded all lumbar and pelvic angle data. After completion of pre-test data collection, all iPhones were removed, and body landmarks were erased.

The participants in the control group were instructed to wait for $15 \mathrm{~min}$, whereas participants in the exercise group underwent a 30-min MCE. After the participants in the control group had finished the 15-min rest, and the participants in the exercise group had completed Motor control exercise, they underwent practice trials and post-test data collection using the same protocol starting with marking all body landmarks.

Each participant was randomly assigned into either the control or exercise group by using a simple random number generator ( $1=$ control group, and $2=$ exercise group) after pre-test data collection. This randomization process was handled by only one 
Table 1. Demographic data at baseline

\begin{tabular}{lcc}
\hline & Control & Exercise \\
\hline Age (years)* & $20.4 \pm 1.4$ & $21.3 \pm 1.1$ \\
Gender & 6.7 & \\
$\quad$ Male (\%) & 26.7 \\
BMI $\left(\mathrm{kg} / \mathrm{m}^{2}\right)$ & $21.0 \pm 2.7$ & $21.2 \pm 2.4$ \\
Lumbar range of motion (degrees) & $34.7 \pm 8.3$ & $33.9 \pm 10.9$ \\
AE30 & $6.2 \pm 5.4$ & $6.8 \pm 4.7$ \\
AE45 & $5.2 \pm 3.7$ & $6.5 \pm 3.2$ \\
AE60 & $4.7 \pm 2.6$ & $6.2 \pm 3.9$ \\
\hline
\end{tabular}

$\mathrm{AE}_{30}$ : Absolute error at angle of $30^{\circ} ; \mathrm{AE}_{45}$ : Absolute error at angle of $45^{\circ}$;

$\mathrm{AE}_{60}$ : Absolute error at angle of $60^{\circ}$.

*Significant difference $(\mathrm{p}<0.05)$.

independent researcher who was not involved in the recruitment process. Each participant received a sealed envelope for group assignment. The control group was provided a 15-min rest by lying on the treatment bed for 15 min, whereas the exercise group underwent a 30-min of MCE based on the motor-learning model ${ }^{17}{ }^{18}$. Due to the nature of the intervention study, we were unable to blind neither participant nor physical therapist. However, only the assessor was blinded to the group assignment.

For intervention, the physical therapists would typically prescribe a 30-min MCE program to improve trunk motor control. This exercise focuses on motor control rather than strength, endurance, or cardiovascular system ${ }^{18)}$. Each participant was assessed to determine the ability of the trunk control (stages 1-3). Exercise was performed at the stage that the participant was unable to perform. For example, the participant could hold the co-contraction of deep abdominal and back muscles for $30 \mathrm{~s}$ (stage 1) but was unable to perform co-contraction with leg movement (stage 2). In this case, the participant would begin the MCE using co-contraction with leg movement. The exercise progressed by modifying lever arm and complexity. This exercise is a low-intensity exercise to develop an ability to automatically control co-contraction of deep abdominal and back muscles during functional movement ${ }^{18)}$. Therefore, the possibility of experiencing muscle fatigue was minimal. However, the participants were instructed to perform stretching exercise after the data collection was completed, and to be perform in the following days to prevent delayed-onset muscle soreness.

Absolute repositioning error was a primary outcome measure in the present study. It was defined as the absolute difference between target angle $\left(30^{\circ}, 45^{\circ}\right.$, or $\left.60^{\circ}\right)$ and actual performance. Data from test (first two sets of three repetitions) and retest (last two sets of three repetitions) from the control group were used to determine test-retest (inter-trial) reliability, and SEM (inter-trial) of the protocol. The rating of perceived exertion (RPE) and heart rate were used to monitor each participant throughout the protocol to avoid fatigue. Pre- and post-test data from the control and exercise groups were used to determine the immediate effect of MCE.

All statistical analyses were performed using SPSS software (IBM SPSS Statistics for Windows, Version 21.0. Armonk, NY, USA). To determine the test-retest reliability of an iPhone application measurement and protocol, the mean angular displacement data from the iPhone application was used to calculate the test-retest reliability by using intraclass correlation coefficient $\left(\mathrm{ICC}_{2, \mathrm{k}}\right)$ with confidence intervals $(\mathrm{CI})$. SEM was also calculated. Descriptive statistics was performed, and statistical assumptions were tested before performing the main statistical analyses. The Kolmogorov-Smirnov test was used to determine data normality. The square root was used to transform data when normality test was violated. A mixed design analysis of variance was used to determine the effects of 30-min MCE on lumbar position sense at $30^{\circ}, 45^{\circ}$, and $60^{\circ}$. This statistical analysis was based on the principles of intention to treat. The significance level will be considered at 0.05 for all analyses. Post-hoc power analyses were also performed.

\section{RESULTS}

The ICC revealed excellent test-retest reliability of an iPhone application $\left(\mathrm{ICC}_{2, \mathrm{k}}=0.95 ; \mathrm{CI}=0.92-0.97\right)$, and good testretest reliability of our protocol $\left(\mathrm{ICC}_{2, \mathrm{k}}=0.67 ; \mathrm{CI}=0.31-0.84\right)$. The SEM for an iPhone application measurement and our protocol was $1.96^{\circ}$ and $4.43^{\circ}$, respectively. Demographic data (Table 1) showed that the two groups were significantly different in age and gender. However, statistical analysis showed that both groups had similar lumbar range of motion and absolute repositioning errors at baseline.

The results demonstrated significant interaction effect of Angle*Time $\left(\mathrm{F}_{2,116}=4.85, \mathrm{p}<0.05, \eta^{2}=0.08\right)$, main effect of Angle $\left(F_{2,116}=10.60, p<0.001, \eta^{2}=0.15\right)$, and main effect of Time $\left(F_{1,58}=4.83, p<0.05, \eta^{2}=0.08\right)$. Post-hoc simple comparisons (Table 2) showed no significant difference between the control and exercise groups at each time point in each angle. However, the exercise group showed significant reduction in absolute repositioning errors $(\mathrm{p}<0.05)$ between pre- and post-test at $45^{\circ}$ and $60^{\circ}$, whereas no significant change was found in the control group. In addition, both groups showed significantly less absolute error $(\mathrm{p}<0.05)$ at $45^{\circ}$ and $60^{\circ}$ comparing with $30^{\circ}$ at post-test, whereas no significant difference was found among angles in the pre-test for both groups. 
Table 2. Post-hoc simple comparisons among groups (Control vs. Exercise), times (Pre vs. Post), and angles $\left(30^{\circ}\right.$ vs. $45^{\circ}$ vs. $60^{\circ}$ )

\begin{tabular}{|c|c|c|c|c|c|c|c|}
\hline & & Mean $\mathrm{AE}_{30} \pm \mathrm{SEM}$ & Mean $\mathrm{AE}_{45} \pm \mathrm{SEM}$ & Mean $\mathrm{AE}_{60} \pm \mathrm{SEM}$ & $\mathrm{AE}_{30-45}[\mathrm{CI}]$ & $\mathrm{AE}_{30-60}[\mathrm{CI}]$ & $\mathrm{AE}_{45-60}[\mathrm{CI}]$ \\
\hline \multirow{3}{*}{ Control } & Control_Pre & $6.22 \pm 0.99$ & $5.17 \pm 0.68$ & $4.72 \pm 0.47$ & $1.05[-0.60,2.71]$ & $1.5[-0.78,3.78]$ & $0.45[-1.14,2.03]$ \\
\hline & Control_Post & $7.26 \pm 1.17$ & $4.57 \pm 0.68$ & $4.17 \pm 0.53$ & $2.69 *[1.12,4.26]$ & $3.09 *[1.19,4.99]$ & $0.4[-0.91,1.71]$ \\
\hline & $\mathrm{AE}_{\text {Pre-Post }}[\mathrm{CI}]$ & $-1.03[-3.19,1.12]$ & $0.6[-0.88,2.08]$ & $0.56[-1.15,2.26]$ & & & \\
\hline \multirow{3}{*}{ Exercise } & Exercise_Pre & $6.83 \pm 0.85$ & $6.52 \pm 0.58$ & $6.28 \pm 0.70$ & $0.31[-1.34,1.97]$ & $0.56[-1.72,2.84]$ & $0.24[-1.34,1.83]$ \\
\hline & Exercise_Post & $7.12 \pm 0.74$ & $4.47 \pm 0.73$ & $3.86 \pm 0.53$ & $2.66^{*}[1.09,4.22]$ & $3.27 *[1.37,5.16]$ & $0.61[-0.70,1.92]$ \\
\hline & $\mathrm{AE}_{\text {Pre-Post }}[\mathrm{CI}]$ & $-0.29[-2.45,1.87]$ & $2.06^{*}[0.57,3.54]$ & $2.42 *[0.72,4.13]$ & & & \\
\hline \multirow{2}{*}{\multicolumn{2}{|c|}{$\begin{array}{l}\mathrm{AE}_{\text {Control-Exercise }} \text { at Pre }[\mathrm{CI}] \\
\mathrm{AE}_{\text {Control-Exercise }} \text { at Post }[\mathrm{CI}]\end{array}$}} & $-0.61[-3.22,1.99]$ & $-1.35[-3.13,0.43]$ & $-1.56[3.25,0.14]$ & & & \\
\hline & & $0.13[-2.63,2.90]$ & $0.1[-1.90,2.10]$ & $0.31[-1.19,1.82]$ & & & \\
\hline
\end{tabular}

$\mathrm{AE}_{30}$ : Absolute error at angle of $30^{\circ} ; \mathrm{AE}_{45}$ : Absolute error at angle of $45^{\circ} ; \mathrm{AE}_{60}$ : Absolute error at angle of $60^{\circ} ; \mathrm{AE}_{30-45}$ : Absolute error difference between $30^{\circ}$ and $45^{\circ} ; \mathrm{AE}_{30-60}$ : Absolute error difference between $30^{\circ}$ and $60^{\circ} ; \mathrm{AE}_{45-60}$ : Absolute error difference between $45^{\circ}$ and $60^{\circ} ; \mathrm{AE}_{\text {Pre-Post }}$ : Absolute error difference between pre and post; $\mathrm{AE}_{\text {Control-Exercise: Absolute error difference between control and }}$ exercise groups; SEM: Standard error of the mean; CI: 95\% confidence interval for difference.

*Significant difference $(\mathrm{p}<0.05)$.

\section{DISCUSSION}

The test-retest reliability of an iPhone application and our protocol showed good to excellent reliability; therefore, we can confidently interpret our data as a result of the effect of MCE. The main outcomes showed that one-session MCE significantly improved the lumbar position sense in lumbar flexion at $45^{\circ}$ and $60^{\circ}$ in the exercise group, whereas no significant change was found between pre- and post-tests in the control group.

MCE significantly improved the lumbar position sense by $31.44 \%$ and $38.54 \%$ reduction in absolute repositioning error at $45^{\circ}$ and $60^{\circ}$, respectively. Our MCE was based on the motor-learning principle to induce repeated intentional muscle contractions and greater muscle recruitment using various self-perturbations ${ }^{17}{ }^{18)}$. Accordingly, the MCE would improve afferent signals from muscle spindle activity ${ }^{6,21)}$. It is generally accepted that primary afferent fibers innervating muscle spindles mainly contribute to the sense of position ${ }^{1)}$. The accuracy of the sensory integration is thus increased as muscle spindle activity increases, inducing a greater position sense. In addition, postural muscles, such as postvertebral muscle, have been presumed to have a relatively high density of muscle spindles ${ }^{27)}$. This evidence would explain a greater position sense after MCE.

The MCE focusing on motor learning may be more appropriate for patients with LBP as opposed to emphasizing trunk strength to stabilize the lumbar spine. Although Kong et al. found joint position sense improvement in patients with chronic LBP, their exercise might not be suitable because those patients were susceptible to fatigability ${ }^{14}$, 28). Joint position sense has been shown to be altered by exercise $\mathrm{e}^{29-31)}$. Different types of muscle contraction, such as concentric, isometric, and eccentric, if performed to fatigue level, could impair the joint position sense ${ }^{1)}$. However, moderate exercise and long-term training induced greater joint position sense accuracy ${ }^{14,30)}$. In the present study, the participants underwent one session of specific MCE. Although, a load was added by moving the upper and/or lower extremities, the participants were instructed to perform the exercise at the non-fatigue level monitored by using RPE, and adequate rest period was also provided after each set of exercise.

We were able to detect improvement in lumbar position sense after MCE. This result supports our insight in that lumbar position sense assessment should consider the interaction among passive osteoligamentous, active muscular, and neural control subsystems ${ }^{7}$. In addition, our result could be used to explain why Boucher et al. did not find a significant change when using the motion perception threshold in which passive subsystem was assessed $\left.{ }^{7}, 13\right)$.

In our experiments, all participants performed at three different angles $\left(30^{\circ}, 45^{\circ}\right.$, and $\left.60^{\circ}\right)$ of lumbar flexion. In the exercise group, significant changes of position sense were found at $45^{\circ}$ and $60^{\circ}$, whereas it was unchanged in lumbar flexion at $30^{\circ}$ compared between pre- and post-test. In addition, both groups showed significantly less absolute repositioning error at $45^{\circ}$ and $60^{\circ}$ compared with $30^{\circ}$ at post-test. The muscle spindles are stretch receptors. When the muscle is lengthened, the discharge rate of muscle spindle afferents increases as the length increases. Therefore, lumbar position sense can be easily perceived at greater degree of lumbar flexion ${ }^{1}$. Solomonow et al. have presented that stress in other structures of the low back, such as spinal ligaments and facet capsule, induces a reflex response that provides a proprioceptive feedback ${ }^{32}$. The sensory inputs from the ligaments and joint capsule are thought to act as limit detector, providing sensory feedback particularly near the end or at the extreme of range of motion ${ }^{32,33)}$. Low back skin receptor has also been reported to contribute to the lumbar position sense ${ }^{34,35}$. In the present study, the contribution from muscles, ligaments, joint capsules, or skin at $30^{\circ}$ of lumbar flexion might be insufficient to enable a greater position sense even with exercise. This would imply that exercise could help improve position sense. However, at a small angle where the structures contributing to position sense are less stretched, the change may not be present. At greater lumbar flexion $\left(45^{\circ}\right.$ and $\left.60^{\circ}\right)$, the repositioning error was significantly less compared with $30^{\circ}$ at post-test in both groups. At pre-test, the same trend was found for both groups even no significant differences among angles were found. This is in line with the study of Wilson et al. that reported less position error in highly flexed position $\left(60^{\circ}\right)$. They proposed that at highly flexed position, the ligamentous structures of the low back begin to 
provide the information on position sense.

In addition, a positive trend was found although the control group did not demonstrate significant improvement in position sense compared pre- and post-test. This may be caused by the learning effect of testing protocol. If yes, this would be also considered for the exercise group. However, with good test-retest reliability of our protocol, we are confident that the greater position sense found in the exercise group was mainly from the exercise. Finally, a significant difference was found in age and gender between the two groups; however, both groups have similar lumbar range of motion and absolute error at three different angles at baseline.

The findings of this study should be considered in light of the following limitations. We used a sample of healthy individuals to investigate the effect of MCE on position sense. This will limit generalizability of our study; therefore, investigation in other population is required. The study design of the present study is a randomized controlled trial; the difference between the two groups is not eliminated.

An iPhone application can be used to investigate lumbar position sense, particularly in the clinical practice to quickly collect data because it is easy to use, low-cost compared with high-precision equipment and do not require other equipment. One session of specific MCE can improve lumbar position sense at high lumbar flexion. This study provides evidence to support the feasibility to further investigate the effect of MCE on position sense in the different group of participants, such as patients with LBP. Moreover, further study would address the long-term effect of this exercise and also concern its influence on differences on lumbar angles.

\section{Funding}

This study was funded in part by Thailand Research Fund (TRG5880133, 2015) and Mahidol University Talent Management Program (Wattananon).

\section{Conflict of interest}

There is no conflict of interest.

\section{ACKNOWLEDGEMENT}

The authors would like to thank the Faculty of Physical Therapy, Mahidol University for providing space for data collection.

\section{REFERENCES}

1) Proske U, Gandevia SC: The proprioceptive senses: their roles in signaling body shape, body position and movement, and muscle force. Physiol Rev, 2012, 92: 1651-1697. [Medline] [CrossRef]

2) Descarreaux M, Blouin JS, Teasdale N: Repositioning accuracy and movement parameters in low back pain subjects and healthy control subjects. Eur Spine J, 2005, 14: 185-191. [Medline] [CrossRef]

3) Newcomer K, Laskowski ER, Yu B, et al.: Repositioning error in low back pain. Comparing trunk repositioning error in subjects with chronic low back pain and control subjects. Spine, 2000, 25: 245-250. [Medline] [CrossRef]

4) O'Sullivan PB, Burnett A, Floyd AN, et al.: Lumbar repositioning deficit in a specific low back pain population. Spine, 2003, 28: 1074-1079. [Medline] [CrossRef]

5) Silfies SP, Cholewicki J, Radebold A: The effects of visual input on postural control of the lumbar spine in unstable sitting. Hum Mov Sci, 2003, 22: 237-252. [Medline] [CrossRef]

6) Wong JD, Wilson ET, Gribble PL: Spatially selective enhancement of proprioceptive acuity following motor learning. J Neurophysiol, 2011, 105: 2512-2521. [Medline] [CrossRef]

7) Panjabi MM: Clinical spinal instability and low back pain. J Electromyogr Kinesiol, 2003, 13: 371-379. [Medline] [CrossRef]

8) Dankaerts W, O'Sullivan P: The validity of O'Sullivan's classification system (CS) for a sub-group of NS-CLBP with motor control impairment (MCI): overview of a series of studies and review of the literature. Man Ther, 2011, 16: 9-14. [Medline] [CrossRef]

9) Rabin A, Shashua A, Pizem K, et al.: A clinical prediction rule to identify patients with low back pain who are likely to experience short-term success following lumbar stabilization exercises: a randomized controlled validation study. J Orthop Sports Phys Ther, 2014, 44: 6-B13. [Medline] [CrossRef]

10) Van Dillen LR, Sahrmann SA, Wagner JM: Classification, intervention, and outcomes for a person with lumbar rotation with flexion syndrome. Phys Ther, 2005, 85: 336-351. [Medline]

11) Teyhen DS, Flynn TW, Childs JD, et al.: Fluoroscopic video to identify aberrant lumbar motion. Spine, 2007, 32: E220-E229. [Medline] [CrossRef]

12) Claus AP, Hides JA, Moseley GL, et al.: Different ways to balance the spine: subtle changes in sagittal spinal curves affect regional muscle activity. Spine, 2009, 34: E208-E214. [Medline] [CrossRef]

13) Boucher JA, Preuss R, Henry SM, et al.: The effects of an 8-week stabilization exercise program on lumbar movement sense in patients with low back pain. BMC Musculoskelet Disord, 2016, 17: 23. [Medline] [CrossRef]

14) Kong YS, Jang GU, Park S: The effects of prone bridge exercise on the Oswestry disability index and proprioception of patients with chronic low back pain. J Phys Ther Sci, 2015, 27: 2749-2752. [Medline] [CrossRef] 
15) Beneck GJ, Story JW, Donald S: Postural cueing to increase lumbar lordosis increases lumbar multifidus activation during trunk stabilization exercises: electromyographic assessment using intramuscular electrodes. J Orthop Sports Phys Ther, 2016, 46: 293-299. [Medline] [CrossRef]

16) Hides JA, Stanton WR, McMahon S, et al.: Effect of stabilization training on multifidus muscle cross-sectional area among young elite cricketers with low back pain. J Orthop Sports Phys Ther, 2008, 38: 101-108. [Medline] [CrossRef]

17) Hodges PW, Moseley GL: Pain and motor control of the lumbopelvic region: effect and possible mechanisms. J Electromyogr Kinesiol, 2003, 13: 361-370. [Medline] [CrossRef]

18) O'Sullivan PB: Lumbar segmental 'instability': clinical presentation and specific stabilizing exercise management. Man Ther, 2000, 5: 2-12. [Medline] [CrossRef]

19) Rackwitz B, de Bie R, Limm H, et al.: Segmental stabilizing exercises and low back pain. What is the evidence? A systematic review of randomized controlled trials. Clin Rehabil, 2006, 20: 553-567. [Medline] [CrossRef]

20) Smith BE, Littlewood C, May S: An update of stabilisation exercises for low back pain: a systematic review with meta-analysis. BMC Musculoskelet Disord, 2014, 15: 416. [Medline] [CrossRef]

21) Hospod V, Aimonetti JM, Roll JP, et al.: Changes in human muscle spindle sensitivity during a proprioceptive attention task. J Neurosci, 2007, 27: 5172-5178. [Medline] [CrossRef]

22) Ostry DJ, Darainy M, Mattar AA, et al.: Somatosensory plasticity and motor learning. J Neurosci, 2010, 30: 5384-5393. [Medline] [CrossRef]

23) Krause DA, Boyd MS, Hager AN, et al.: Reliability and accuracy of a goniometer mobile device application for video measurement of the functional movement screen deep squat test. Int J Sports Phys Ther, 2015, 10: 37-44. [Medline]

24) Tousignant-Laflamme Y, Boutin N, Dion AM, et al.: Reliability and criterion validity of two applications of the iPhone ${ }^{\mathrm{TM}}$ to measure cervical range of motion in healthy participants. J Neuroeng Rehabil, 2013, 10: 69. [Medline] [CrossRef]

25) Cholewicki J, Polzhofer GK, Radebold A: Postural control of trunk during unstable sitting. J Biomech, 2000, 33: 1733-1737. [Medline] [CrossRef]

26) Mok NW, Brauer SG, Hodges PW: Failure to use movement in postural strategies leads to increased spinal displacement in low back pain. Spine, 2007, 32: E537-E543. [Medline] [CrossRef]

27) Amonoo-Kuofi HS: The number and distribution of muscle spindles in human intrinsic postvertebral muscles. J Anat, 1982, 135: 585-599. [Medline]

28) Larivière C, Bilodeau M, Forget R, et al.: Poor back muscle endurance is related to pain catastrophizing in patients with chronic low back pain. Spine, 2010, 35: E1178-E1186. [Medline] [CrossRef]

29) Allen TJ, Leung M, Proske U: The effect of fatigue from exercise on human limb position sense. J Physiol, 2010, 588: 1369-1377. [Medline] [CrossRef]

30) Bartlett MJ, Warren PJ: Effect of warming up on knee proprioception before sporting activity. Br J Sports Med, 2002, 36: 132-134. [Medline] [CrossRef]

31) Taimela S, Kankaanpää M, Luoto S: The effect of lumbar fatigue on the ability to sense a change in lumbar position. A controlled study. Spine, 1999, 24: 1322-1327. [Medline] [CrossRef]

32) Solomonow M, Zhou BH, Harris M, et al.: The ligamento-muscular stabilizing system of the spine. Spine, 1998, 23: 2552-2562. [Medline] [CrossRef]

33) Janwantanakul P, Magarey ME, Jones MA, et al.: Variation in shoulder position sense at mid and extreme range of motion. Arch Phys Med Rehabil, 2001, 82: 840-844. [Medline] [CrossRef]

34) Beaudette SM, Larson KJ, Larson DJ, et al.: Low back skin sensitivity has minimal impact on active lumbar spine proprioception and stability in healthy adults. Exp Brain Res, 2016, 234: 2215-2226. [Medline] [CrossRef]

35) Hidalgo B, Gobert F, Bragard D, et al.: Effects of proprioceptive disruption on lumbar spine repositioning error in a trunk forward bending task. J Back Musculoskeletal Rehabil, 2013, 26: 381-387. [Medline] [CrossRef] 